\title{
Asg1 is a stress-inducible gene which increases stomatal resistance in salt stressed potato
}

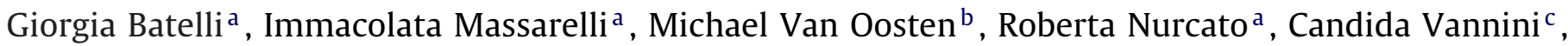 \\ Giampaolo Raimondi ${ }^{\mathrm{b}}$, Antonella Leone ${ }^{\mathrm{d}}$, Jian-Kang Zhu ${ }^{\mathrm{e}}$, Albino Maggio ${ }^{\mathrm{b}}$, Stefania Grillo ${ }^{\mathrm{a}, *}$ \\ a CNR Institute of Plant Genetics (CNR-IGV), Via Universita', 13380055 Portici, Italy \\ b Department of Agricultural Engineering and Agronomy, University of Naples Federico II, Via Università 100, 80055 Portici, Italy

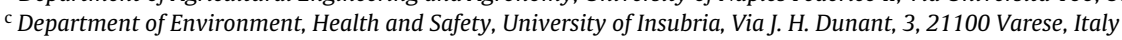 \\ ${ }^{\mathrm{d}}$ Department of Pharmaceutical and Biomedical Sciences, University of Salerno, Via Ponte Don Melillo, 84084 Fisciano, Italy \\ e Department of Horticulture and Landscape Architecture, Purdue University, 47907 West Lafayette, IN, USA
}

\section{A R T I C L E I N F O}

\section{Article history:}

Received 16 May 2012

Received in revised form 6 July 2012

Accepted 7 July 2012

\section{Keywords:}

Arabidopsis

Germination

Salt stress

Stomata resistance

Potato

\begin{abstract}
A B S T R A C T
The identification of critical components in plant salt stress adaptation has greatly benefitted, in the last two decades, from fundamental discoveries in Arabidopsis and close model systems. Nevertheless, this approach has also highlighted a non-complete overlap between stress tolerance mechanisms in Arabidopsis and agricultural crops. Within a long-running research program aimed at identifying salt stress genetic determinants in potato by functional screening in Escherichia coli, we isolated Asg1, a stress-related gene with an unknown function. Asg1 is induced by salt stress in both potato and Arabidopsis and by abscisic acid in Arabidopsis. Asg1 is actively transcribed in all plant tissues. Furthermore, Asg1 promoter analysis confirmed its ubiquitous expression, which was remarkable in pollen, a plant tissue that undergoes drastic dehydration/hydration processes. Fusion of Asg1 with green fluorescent protein showed that the encoded protein is localized close to the plasma membrane with a non-continuous pattern of distribution. In addition, Arabidopsis knockout asg1 mutants were insensitive to both $\mathrm{NaCl}$ and sugar hyperosmotic environments during seed germination. Transgenic potato plants over-expressing the Asg1 gene revealed a stomatal hypersensitivity to $\mathrm{NaCl}$ stress which, however, did not result in a significantly improved tuber yield in stress conditions. Altogether, these data suggest that Asg1 might interfere with components of the stress signaling pathway by promoting stomatal closure and participating in stress adaptation.
\end{abstract}

(c) 2012 Elsevier GmbH. All rights reserved.

\section{Introduction}

Salt stress is one of the major constraints to agriculture, causing large yield decreases in a variety of crops all over the world (Munns, 2002; Flowers, 2004). High concentrations of salt in the soil are known to affect both ionic and osmotic homeostasis in plants, causing reduction of leaf transpiration, inhibition of shoot growth and decreased seed germination (Zhu, 2002; Munns and Tester, 2008). Considerable progress has been made over the last two decades toward the elucidation of fundamental mechanisms underlying plant stress tolerance with the ultimate goal of identifying candidate traits to improve the performance of agricultural crops (Atkinson and Urwin, 2012). Key players in the complex network

\footnotetext{
Abbreviations: ABA, abscisic acid; GFP, green fluorescent protein.

* Corresponding author at: National Research Council, Institute of Plant Genetics (CNR-IGV), Via Universita', 13380055 Portici (NA), Italy. Tel.: +39 081 2539213/2539205; fax: +39 0817753579

E-mail address: grillo@unina.it (S. Grillo).
}

of stress induced responses have been identified (Shinozaki and Yamaguchi-Shinozaki, 2007; Krasensky and Jonak, 2012). These include components of the ion homeostasis machinery (Amtmann and Leigh, 2010), ROS signaling and scavenging systems (Foyer and Shigeoka, 2011), water movements and osmoregulation (Pardo, 2010; Verslues and Juenger, 2011; Aroca et al., 2012), signaling (Kuromori et al., 2010; Weiner et al., 2010; Lee and Luan, 2012), hormonal regulation (Raghavendra et al., 2010; Klingler et al., 2010). Only a few of these genes, mostly isolated in Arabidopsis through forward genetic screenings, have been successfully transferred into crop species (Nelson et al., 2007; Karaba et al., 2007; Qin et al., 2011; Deikman et al., 2012). This may partially be due to an imperfect overlap of adaptation mechanisms between Arabidopsis and other plants, including agriculturally important species. For example, the accumulation of proline or other compatible solutes is not typical of Arabidopsis, which produces only $20 \mu \mathrm{mol} / \mathrm{g}$ of proline fresh weight in response to $200 \mathrm{mM} \mathrm{NaCl}$ (Tsugane et al., 1999). In contrast, tomato plants subjected to the same level of stress accumulate proline up to $100 \mu \mathrm{mol} / \mathrm{g}$ of fresh weight (Fujita et al., 1998), and they have a constitutive $200 \mu \mathrm{mol} / \mathrm{g}$ of fresh weight of proline 
in pollen. Other mechanisms that lead to salt stress adaptation may differentially promote shoot $v$ s. root development and indirectly affect the commercial portion of the plant. For instance, assessment of plant stress tolerance based on the aboveground plant response to salinity may not be informative for understanding the development of underground commercial organs such as roots (Chołuj et al., 2008) and tubers (Singh and Kaur, 2009).

With the long-term objective of identifying critical components in salt stress adaptation in crop plants, a cDNA library obtained from Solanum commersonii, a stress tolerant wild relative of the cultivated potato, was expressed in Escherichia coli to identify cDNA clones conferring to $E$. coli the ability to grow on high concentrations of salt (Massarelli et al., 2006). E. coli has been shown to be suitable for the identification of downstream components directly involved in plant stress responses and tolerance acquisition. Yamada et al. (2003) isolated from the halophyte Sueda japonica the gene RelA/SpoT, encoding a phosphoethanolamine Nmethyl transferase able to confer salt tolerance to E. coli. Similarly, Arabidopsis cDNAs encoding DRT (DNA-damage-repair/toleration) proteins were isolated by complementation of $E$. coli mutants impaired in the DNA repair mechanisms against UV-light damage (Pang et al., 1993). By overexpressing in E. coli a S. commersonii cDNA library, genes encoding for proteins already known to confer stress tolerance, such as dehydrins and chaperonins were also identified, thus providing further evidence that functional screenings in E. coli are suitable for the isolation of genes directly involved in the cellular responses to salt stress (Massarelli et al., 2006).

One of the identified potato cDNA clones, N2.1, encoded a previously uncharacterized gene, named ASG1 (Abiotic Stress Gene 1). Asg1 encodes a putative protein of unknown function with a predicted DUF1005 domain of unknown function at the C-terminus which is shared among members of a small plant-specific protein family. Although Asg1 has no sequence similarities that could suggest its biological function in plant cells, its isolation through a functional screening indicated that Asg1 could contribute to plant stress response mechanisms. In this study, by a transcriptional and functional analysis of Asg1 gene in response to stress conditions, we demonstrate that Asg1 is a positive regulator of osmotic stress response in Solanum tuberosum and Arabidopsis thaliana via an abscisic acid (ABA)-dependent pathway.

\section{Materials and methods}

\section{Plant material}

Solanum tuberosum cv. Desirèe was the wild-type background genotype for potato experiments. Plants propagated from internodes and grown in vitro for 4 weeks on MS30 medium (Murashige and Skoog, 1962) prior to transfer in vivo were used for physiological and expression analyses.

The Arabidopsis ecotype Col-0 was used as wild-type control. Seeds of the T-DNA insertion mutant for Asg1 (asg1, Salk_059272) were obtained from the Arabidopsis Biological Resource Center (ABRC). Homozygous knockout mutants were selected by PCR using primers LP and RP.

\section{Growth conditions and stress treatments}

\section{Potato}

Different experimental systems were used to study potato response to salt stress. Hydroponics cultures, obtained from in vitro grown plants transferred to solution containing $0.4 \times$ MS were exposed to $200 \mathrm{mM} \mathrm{NaCl}$ for gene expression analyses or to $100 \mathrm{mM}$ $\mathrm{NaCl}$ for stomatal resistance and water potential studies. For measurements of physiological and yield parameters in the presence of salt stress, in vitro-propagated plants transferred to pots containing a mix peat:perlite $=1: 1$ were used. Plants were daily irrigated with Hoagland nutrient solution.

For a prolonged salt stress experiment, potato plants from transgenic line (15A8) and controls (wild type and vector only) were grown in greenhouse and exposed to 3 salt concentrations (50, 100 and $150 \mathrm{mM} \mathrm{NaCl}$ ). Salinization was accomplished by adding weekly increments of $50 \mathrm{mM} \mathrm{NaCl}$ to the nutrient solution. After three weeks, three salinity levels were reached plus a non-salinized control. Six weeks after the initial transfer of the plants to soil, physiological (stomatal resistance and leaf water potential), biometric (total biomass and leaf area) and productive data (tubers fresh weight) were collected. For short-term salt stress experiments, plants were grown in growth chambers in controlled conditions of temperature $\left(20^{\circ} \mathrm{C}\right.$ Day, $15^{\circ} \mathrm{C}$ Night), relative humidity $(50 \%)$ and photoperiod $(12 \mathrm{~h}$ ). One week after transfer to pots 36 plants ( 6 per genotype) were randomly arranged and irrigated with Hoagland solution or Hoagland solution plus $100 \mathrm{mM} \mathrm{NaCl}$. Stomatal resistance and leaf water potential were measured one week after initiation of salt treatments.

\section{Arabidopsis}

Stress treatments were performed on 2-week-old seedlings grown in MS medium in liquid culture. Seedlings were treated for $3 \mathrm{~h}$ with abscisic acid $100 \mu \mathrm{M}$; Myo-inositol $1 \mathrm{mM}$; LiCl $25 \mathrm{mM}, \mathrm{KCl}$ $25 \mathrm{mM}, \mathrm{CoCl}_{2} 1 \mu \mathrm{M}, \mathrm{CsCl} 18 \mathrm{mM}, \mathrm{CuSO}_{4} 1 \mu \mathrm{M}, \mathrm{MnSO}_{4} 1 \mathrm{mM}, \mathrm{ZnSO}_{4}$ $300 \mu \mathrm{M}$.

Water loss analyses on detached leaves of wild-type, asg1 and T-3 single copy homozygous Asg1 over-expressing plants were performed as described by Verslues et al. (2006), using leaves of similar size detached from 4 -week-old plants. Six replicates were performed for each genotype.

Germination analyses in the presence of various stresses were performed using sterilized seeds sown on plates and stratified at $4^{\circ} \mathrm{C}$ for 2 days.

\section{Physiological analyses}

Stomatal resistance measurements were performed on fully expanded leaves of transgenic and control plants using a porometer (AP4, delta device). Leaf water potential measurements were performed on the first fully expanded leaf using a dew-point psychrometer (WP4, Decagon Devices, Pullman, WA). Leaf area was measured on green leaves using a Li-Cor 3000 area meter (Li-Cor, Lincoln, NE).

\section{Gene expression analyses}

Total RNA was isolated from various tissues of potato and Arabidopsis using the TRIZOL reagent (Invitrogen). cDNA was synthesized starting from $1 \mu \mathrm{g}$ of DNase-treated total RNA using Superscript reverse transcriptase (Invitrogen). $2 \mu$ l of cDNA were used for semi-quantitative RT-PCR. Primers used are listed in Table S1. For amplification of Asg1 primers were N2.1 ST1 and N2.1 ST2 for potato andAtN2.1 For and AtN2.1 Rev for Arabidopsis. For normalization of the RT-PCRs $18 \mathrm{~S}$ rRNA was used as internal control for potato (primers 18S1 and 18S2), while for Arabidopsis 18S rRNA (18S1 and 18S2 At), or Actin2 (Primers Act For and Act Rev) were used.

\section{Production of transgenic plants}

Primers used for DNA amplification during cloning steps are listed in Table S1. To obtain potato transgenic plants overexpressing ScAsg1, the coding sequence of ScAsg1 was cloned in 
the binary vector pKYLX71 35S² (Schardl et al., 1987). ScAsg1 was amplified (Primers N2.1 ATG and N2.1 STOP) from the cDNA clone AM050808 and cloned in Xhol-digested pKYLX71 35 S2 after fill-in. The insertion of the fragment in the correct direction was checked by PCR.

Potato internodes from tissue cultured potato plantlets were infected and co-cultivated with Agrobacterium tumefaciens strain LBA4404 carrying the plasmid Asg1::pKYLX71. Several independent $\operatorname{Kan}^{\mathrm{r}}$ plants were regenerated following the method described by De Palma et al. (2008) and further characterized by PCR and semi-quantitative RT-PCR analyses using N2.1ATG and $\mathrm{CH} 11$ primers. To over-express Asg1 in Arabidopsis, the coding sequence of AtAsg1 was amplified from RNA and inserted in pDONR207 (Invitrogen) using the primers AtN2.1DONRFor and AtN2.1DONRevnostop. Asg1 was then transferred to pMDC32 (Curtis and Grossniklaus, 2003) using LR clonase (Invitrogen). For AtAsg1Promoter::GUS expression studies, a fragment of $\sim 2 \mathrm{~Kb}$, corresponding to the putative Arabidopsis Asg1 promoter, was PCR amplified and cloned in pENTR/TOPO (Invitrogen) using primers N2.1 Prom left and N2.1 Prom right. After sequencing, Asg1 promoter was transferred by recombination to PMDC164 vector (Curtis and Grossniklaus, 2003).

Arabidopsis wild-type plants were transformed using the floral dip method (Clough and Bent, 1998) and A. tumefaciens strain GV3101.

\section{GUS staining}

Arabidopsis seedlings and adult plants carrying Asg1Prom::GUS were used for GUS activity measurements. The histochemical detection of GUS activity was performed as previously described (Sunkar et al., 2006). GUS staining patterns were confirmed by observing at least five different transgenic T1 lines, and T2 lines were used for detailed analysis.

\section{Transient expression in onion epidermal cells}

For sub-cellular localization studies, ScN2.1 fragment was inserted in BamHI-digested psm-RSGFP (Davis and Vierstra, 1998) after fill-in. Biolistic bombardments were performed with a PSD1000/He instrument (Bio-Rad). Acceleration of gold microcarriers $(1.6 \mathrm{~lm})$ coated with $1.25 \mathrm{lg}$ of plasmid DNA was used to transform onion epidermal cells. Bombardment parameters were as follows: vacuum, $28 \mathrm{in}$. $\mathrm{Hg}$; distance to target, $6 \mathrm{~cm}$; helium pressure, 650 psi. Onion scales were left for $12-24 \mathrm{~h}$ in the dark at $21^{\circ} \mathrm{C}$, and then epidermal tissues were removed and layered in water on glass slides for microscopy. Confocal microscopy was carried out using a confocal laser-scanning microscope (Bio-Rad MRC 1024 confocal system) equipped with a $\mathrm{Kr}$-Ar laser and mounted on an upright Zeiss Axiovert microscope (Zeiss). Green fluorescent protein was excited at $488 \mathrm{~nm}$; fluorescence was collected between $500 \mathrm{~nm}$ and $535 \mathrm{~nm}$.

\section{Results}

\section{Asg1 is stress-inducible both in potato and Arabidopsis}

The Asg1 gene (Acc. No. AM050808), isolated from the N2.1 clone able to confer salt stress tolerance to $E$. coli, and its putative orthologue in Arabidopsis (At5g17640) were previously shown to be up-regulated after treatments with $\mathrm{NaCl}$ in plants of $A$. thaliana and cells of $S$. tuberosum (Massarelli et al., 2006). To further investigate the possible involvement of Asg1 in the response to stress in plants, the regulation of gene expression in response to stress treatments was assessed by semi-quantitative RT-PCR. RNA blot
(A)

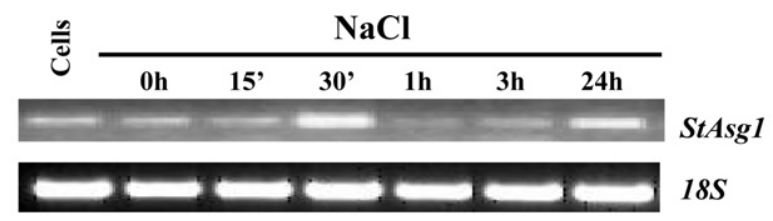

(B)

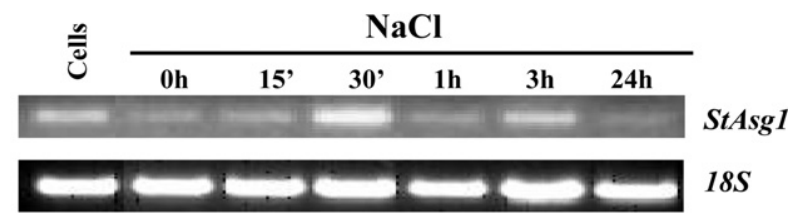

(C)
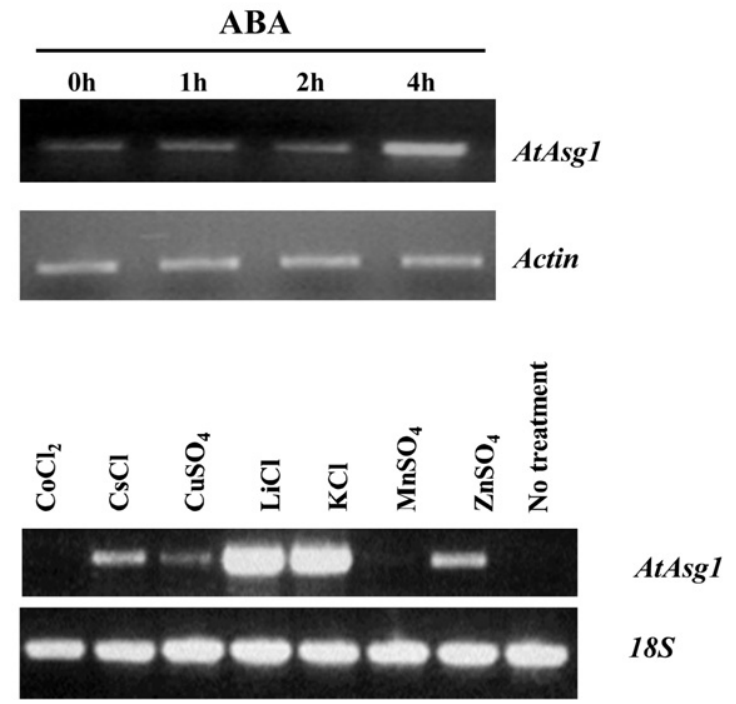

Fig. 1. Expression analysis of the gene Asg1 in response to treatments by semiquantitative RT-PCR. Solanum tuberosum Asg1 response to salt treatments (200 mM $\mathrm{NaCl}$ ) in leaves (A) and roots (B). (C) Time course analysis of Arabidopsis plants treated with ABA $100 \mu \mathrm{M}$; (D) 3 h treatments of Arabidopsis 2 week old seedlings grown in liquid culture and treated with different salts; cells: RNAs from S. tuberosum cell cultures were amplified as controls. 18S ribosomal RNA (A, B and D) and $\beta$-actin (C) were amplified as internal standards.

analysis was attempted under many different conditions but no positive hybridization with Asg1 probe was ever obtained with RNA extracted from wild-type plants of potato and Arabidopsis (data not shown), possibly indicating that the amount of transcript was below the detection limit of this technique. A time course experiment in potato plants treated with $\mathrm{NaCl} 200 \mathrm{mM}$ was performed and semi-quantitative RT-PCR analyses on RNA extracted from roots and leaves were carried out. As shown in Fig. 1, the Asg1 transcripts were rapidly and transiently induced by $\mathrm{NaCl}$, both in leaves (Fig. 1A) and roots (Fig. 1B) of S. tuberosum. The transcript was induced within 30 min of salt treatment while after $1 \mathrm{~h}$ the transcript levels were similar to those of the control samples (Fig. 1A and B).

A time course experiment, carried out exposing Arabidopsis seedlings to ABA for 1-4h showed that the expression of Asg1 gene was considerably induced within $4 \mathrm{~h}$ of treatment with ABA (Fig. 1C). This induction was transient as, after $8 \mathrm{~h}$, the transcript levels were similar to those detected in untreated samples (data not shown).

In a different experiment, liquid cultured seedlings of Arabidopsis were treated for $3 \mathrm{~h}$ with various salts. As shown in Fig. 1D, while $\mathrm{CoCl}_{2}$ and $\mathrm{MnSO}_{4}$ did not affect the expression of $A s g 1$, its expression was induced when plants were treated with $\mathrm{CsCl}$ and $\mathrm{ZnSO}_{4}$ and particularly with $\mathrm{LiCl}$ and $\mathrm{KCl}$. 
(A)

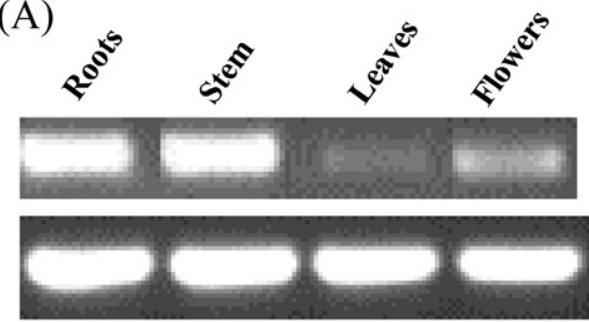

(C)
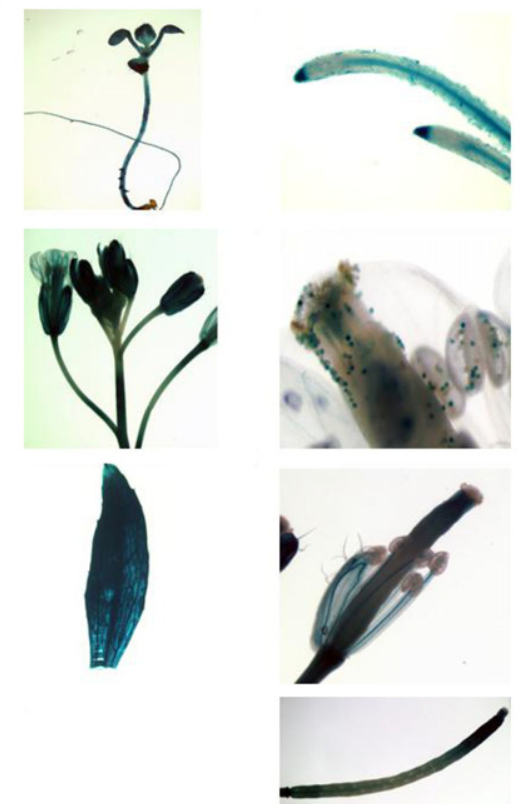

(B)
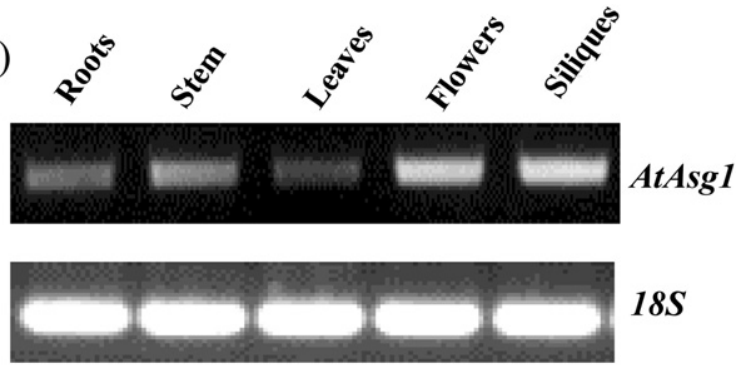

$18 S$

(D)
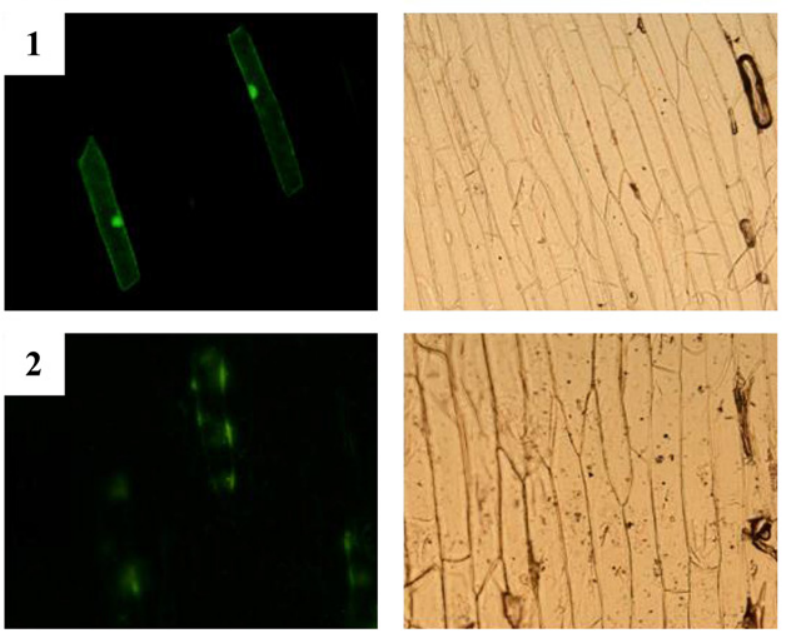

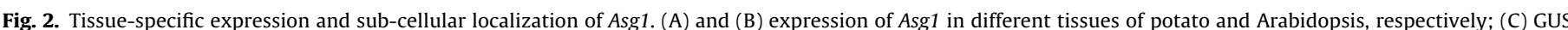

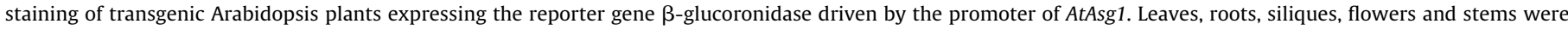

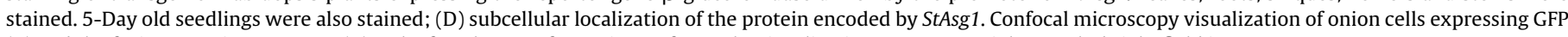
(1) and the fusion protein ASG1-GFP (2) $48 \mathrm{~h}$ after the transformation. Left panels, visualization at $490 \mathrm{~nm}$. Right panels, bright field image.

Taken together, these data indicate that the expression of Asg1 is mainly affected by treatments with salts, such as $\mathrm{NaCl}, \mathrm{LiCl}$ and $\mathrm{KCl}$. The expression of Asg1 is also up-regulated by ABA, which causes a transient induction after $4 \mathrm{~h}$ of treatment.

\section{Asg1 tissue-specific expression and sub-cellular localization}

Understanding the expression pattern and sub-cellular localization of a gene of interest is important to gain insights into its possible function in stress response mechanisms.

In order to study the expression pattern of Asg1, semiquantitative RT-PCRs were carried out on different tissues of Arabidopsis and potato. Asg1 expression was observed in all the analyzed tissues of potato (Fig. 2A) and Arabidopsis (Fig. 2B) plants. The expression of Asg1 was higher in roots and stem of potato plants, while in Arabidopsis the highest level of expression was observed in reproductive tissues. A lower expression of Asg1 compared to other tissues was observed in leaves of both species.

To further analyse the spatial and temporal expression pattern of Asg1 in Arabidopsis, transgenic plants expressing the $\beta$-glucoronidase (GUS) gene driven by the Asg1 putative promoter were obtained.

Analysis of the transgenic plants (Fig. 2C) revealed high GUS activity in all the tissues of young seedlings and in adult plants, particularly in vascular tissues of leaves and roots. The GUS expression pattern was consistent, in general, with the results from semi-quantitative RT-PCR, except that semi-quantitative RT-PCR analyses detected a comparably lower level of expression of Asg1 in leaves despite a high promoter and GUS activity observed in this tissue.

To investigate the sub-cellular localization of the protein encoded by Asg1, a fusion protein ASG1-GFP was transiently expressed in epidermal onion cells and the recombinant protein visualized by confocal microscopy $48 \mathrm{~h}$ after the particle bombardment. As shown in Fig. 2D, while the green fluorescent protein (GFP) was localized as expected in all cellular compartments, the fusion protein ASG1-GFP was mainly localized close to the plasma membrane, with a non-homogeneous and polarized pattern of distribution (Fig. 2D).

Asg1 over-expressing potato plants have a hypersensitive stomatal response to salt stress

ScAsg1 overexpression was previously shown to confer to $E$. coli the ability to grow in the presence of high concentrations of $\mathrm{NaCl}$ (Massarelli et al., 2006). To test whether Asg1 is also able to increase salt tolerance in potato, transgenic potato plants constitutively expressing the S. commersonii Asg1 (ScAsg1) gene under 


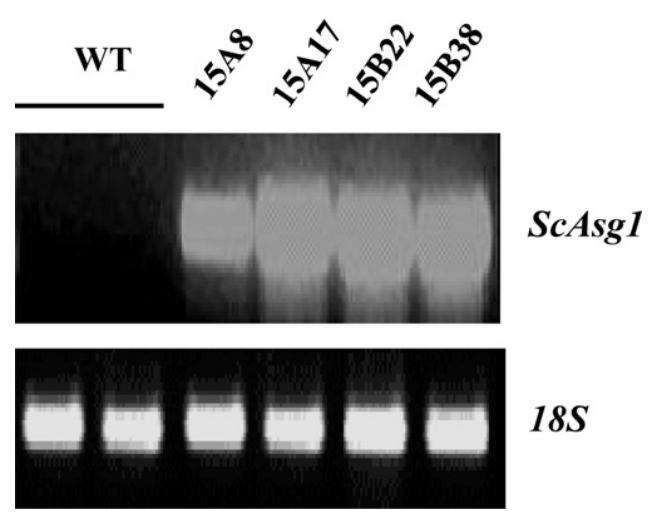

Fig. 3. Expression of the transgene in $S$. tuberosum plants over-expressing the $S$. commersonii gene ScAsg1. Semiquantitative RT-PCR analysis on RNA from leaves of transgenic lines. WT = control untransformed plants (S. tuberosum cv. Desirèe).

the control of the Cauliflower Mosaic Virus $35 S^{2}$ promoter were generated. The expression of ScAsg1 in different lines of potato regenerated after transformation of internodes was confirmed by semi-quantitative RT-PCR (Fig. 3).

Transgenic plants and wild-type potato were subjected to salt stress and physiological parameters measured. Salt stress caused an increase in Stomatal Resistance (SR) and a decrease in total leaf water potential ( $\left.\Psi_{\text {tot }}\right)$ in all the tested plants (Fig. $4 \mathrm{~A}$ and B). ScASG1-overexpressing plants, however, showed a significantly higher SR in the presence of salt stress ranging from 2.6 to $4.3 \mathrm{~s} / \mathrm{cm}$, while in wild-type plants and transformants with the empty vector the SR values ranged between 1.9 and $2.2 \mathrm{~s} / \mathrm{cm}$ (Fig. 4A). In terms of $\Psi_{\text {tot }}$ transgenic lines did not show significant differences when compared with control lines, with the exception of line 15A8, which showed a significantly lower $\Psi_{\text {tot }}$ compared to the controls (Fig. 4B).

To confirm these results, a time course experiment was performed by measuring SR every hour during $11 \mathrm{~h}$ after the imposition of salt stress.

The SR values of plants irrigated with non-salinized solution were similar in ScAsg1 transgenic lines and in the wild-type (Fig. 4C). However, upon salinization the transgenic lines revealed a more rapid increase in stomatal resistance compared to the control plants (Fig. 4D). This increase was not detected in untransformed plants, which on the contrary, seemed somehow relieved from an initial drought stress, possibly because of the 2 days of water withdrawn preceding the time course experiment. SR values were highest in the transgenic line 15A8, in which the average values increased from $8 \mathrm{~s} / \mathrm{cm}$ to $16 \mathrm{~s} / \mathrm{cm}$ (Fig. $4 \mathrm{D}$ ).

Asg1 affects leaf area development in transgenic potato plants exposed to salinity

In a prolonged salt stress experiment, we evaluated whether the anticipated stomatal closure of line $15 \mathrm{~A} 8$ could have been beneficial for plant growth and tuber yield under salt stress. We confirmed the higher stomatal resistance of line 15A8 in response to longterm salt stress induced by irrigation with 100 and $150 \mathrm{mM} \mathrm{NaCl}$ (Fig. 5A), which was not associated to higher ABA levels (Fig. S1). Moreover, line 15A8 showed a higher Leaf Area compared to the controls in salt stress conditions (Fig. 5B). The prompt stomatal closure upon salinization, likely implicated/involved in the facilitated plant adaptation to the hyperosmotic environment and subsequent growth resumption, did not translate in an increased tuber yield in the transgenic line (Fig. 5C).
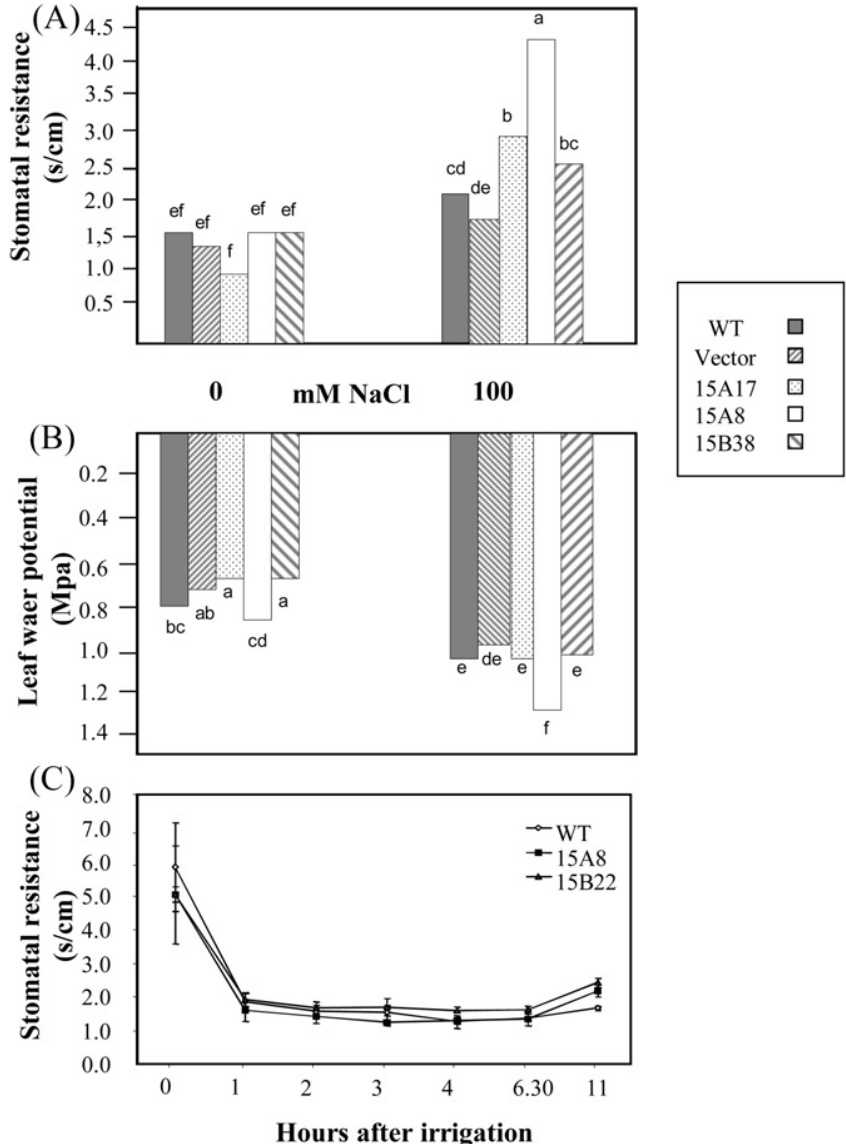

(D)

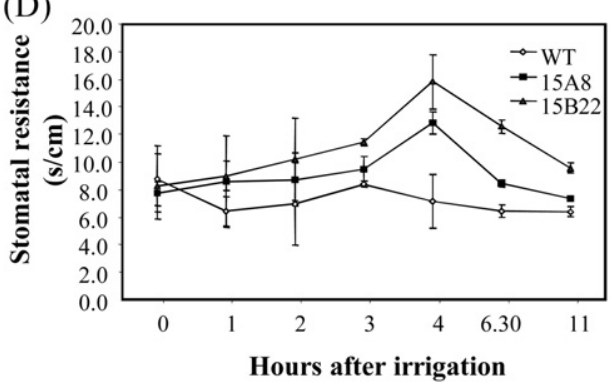

Fig. 4. Effect of ScAsg1 over-expression on stomatal resistance and leaf water potential in salt-stressed plants. Stomatal resistance $(A)$ and leaf water potential (B) of potato transgenic plants over-expressing ScAsg1 (lines 15A17, 15A8, 15B22 and 15B38) grown in hydroponics in control conditions or treated for one week with $100 \mathrm{mM} \mathrm{NaCl}$ in the nutrient media. Untransformed plants (S. tuberosum cv. Desirèe, WT) and plants transformed with the empty vector (Vector) were used as controls. Different letters indicate significant difference at $P<0.05(n=4)$; (C and D) stomatal resistance values of transgenic ScAsg1 over-expressing plants (15A8 and 15B22) and control untransformed plants (WT) watered with nutrient media (C) or nutrient media supplemented with $100 \mathrm{mM} \mathrm{NaCl}$ (D). The time course analysis was performed one week after transfer to pots of in vitro grown plants. Water was withheld for two days before the experiment. Values represent means $\pm S E$ of six replicates.

Seed germination of Arabidopsis asg1 mutant is insensitive to $\mathrm{NaCl}$ and sugars

In order to understand whether a modified expression of Asg1 affects the ability of Arabidopsis to cope with salt or, more in general, with osmotic stress conditions, Asg1 over-expressing and Asg1 knockout (asg1) plants were obtained (Fig. S2) and exposed to saline stress.

Leaf water loss measurements were carried out on Asg1 overexpressing plants as well as on wild-type and asg1 plants as an 

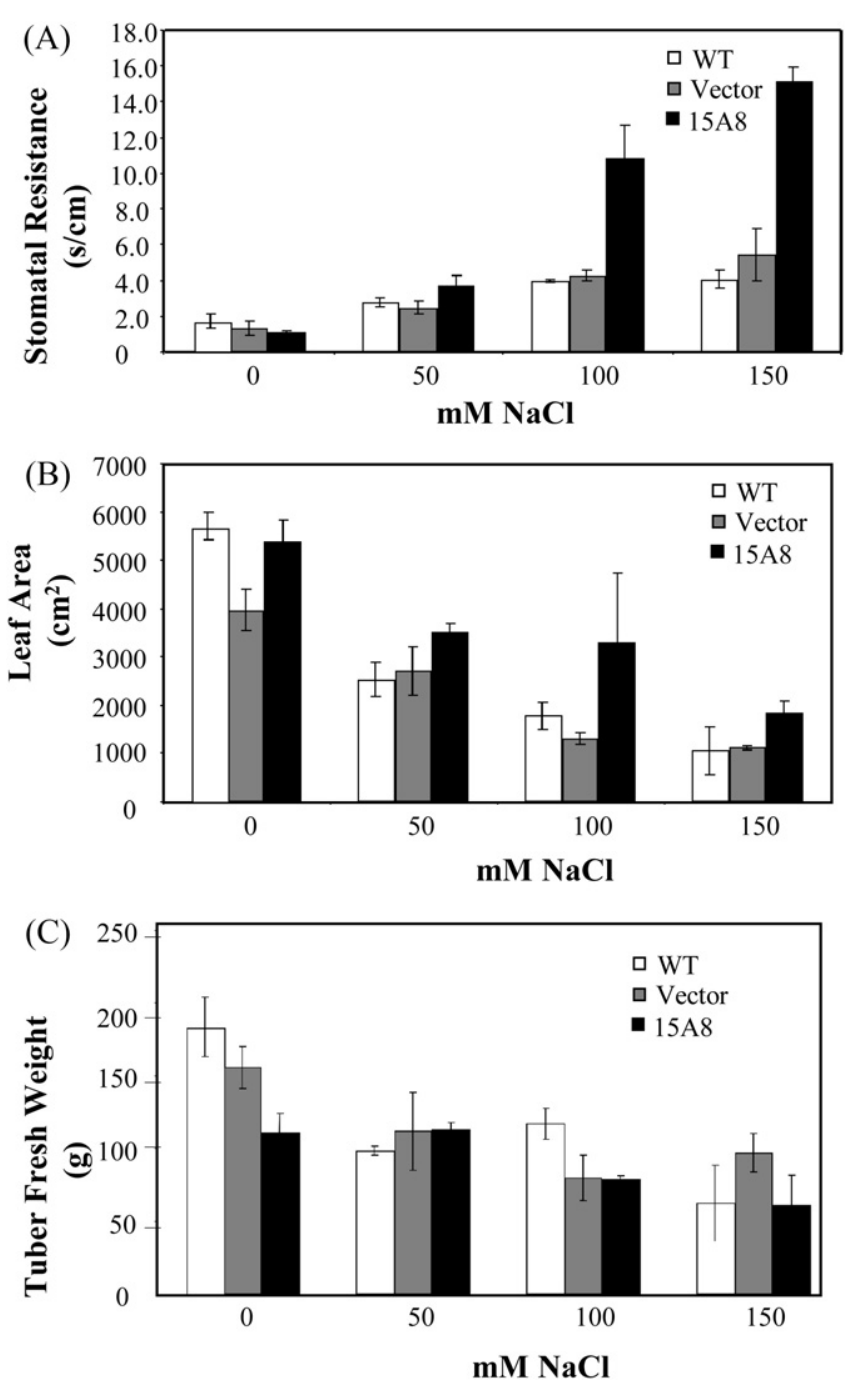

Fig. 5. Impact of salt stress on physiological and productive parameters of ScAsg1 over-expressioning lines. Stomatal resistance (A) and growth parameters (B and C) measured in NaCl-treated potato plants over-expressing ScAsg1 (15A8), control untransformed plants $(\mathrm{WT}=S$. tuberosum $\mathrm{cv}$. Desirèe $)$ and plants transformed with the empty vector (Vector). Plants were grown in greenhouse and subjected to gradually increasing $\mathrm{NaCl}$ treatment $(50 \mathrm{mM}$ increase per week). Data were collected two weeks after the higher salt concentration was reached. (A) Stomatal resistance values; (B) total leaf area; (C) tubers fresh weight. Data are the mean of three replicates.

indirect method of measuring stomatal conductance (Verslues et al., 2006). While asg1 plants did not show any significant differences compared to wild-type, Asg1 overexpressors showed a slightly reduced water loss (Fig. 6), thus suggesting that an increased expression of Asg1 improves, albeit to a small extent, stomatal closure in Arabidopsis.

To analyze the effect of an abolished expression of Asg1 at the germination stage, seed germination was tested in the presence of $\mathrm{NaCl}$ and glucose, which are known to inhibit germination through a mechanism at least in part dependent on ABA (Arenas-Huertero et al., 2000). asg1 was found to be insensitive to $\mathrm{NaCl}$ at the germination stage when germination was scored in terms of radical emergence (Fig. 7A and B). Similar germination tests using ASG1 overexpressors did not show any significant difference in ability to germinate in salt stress conditions as compared to WT (data not shown). asg1 seeds were also insensitive to the addition of glucose or mannose in the medium either when radical emergence was scored and when assays were carried out measuring the emergence of green cotyledons (Fig. 7C and D).

\section{Discussion}

Biological function of ASG1 and its role in plant salt adaptation

We studied the function of Asg1 gene of potato and Arabidopsis using different approaches, including gene expression analysis, overexpression in potato and Arabidopsis and characterization of knockout mutants in Arabidopsis. The Asg1 gene was identified through a functional screening of $S$. commersonii cDNA clones able to confer to E. coli the ability to grow on media containing $0.5 \mathrm{M}$ $\mathrm{NaCl}$ (Massarelli et al., 2006). The gene Asg1 of S. commersonii (ScASG1) encodes a protein of 433 amino acids that does not contain any known functional domain. The ScASG1 protein is $90 \%$ identical to the $S$. tuberosum protein encoded by the Transcript Assembly TA28150_4113. ScASG1 shares high sequence similarity percentages (80\%) with a protein of unknown function of $A$. thaliana (At5g17640) that was hypothesized to be the ASG1 orthologue (AtASG1). In Arabidopsis Asg1 appears to be a unique gene, sharing no high sequence homology with other proteins and containing a large domain of unknown function at the C-terminus, DUF1005, also found in other plant putative or expressed proteins of unknown function. DUF1005 appears to be a plant specific domain. Asg1 was shown to be induced by salt stress either in potato and Arabidopsis but with a different temporal expression pattern. In potato, ASG1 was rapidly yet transiently induced in both leaves and roots after $30^{\prime}$ of exposure to $200 \mathrm{mM} \mathrm{NaCl}$. In contrast, in Arabidopsis, the induction of this gene by $\mathrm{NaCl}$ was also rapid but stable over $24 \mathrm{~h}$ (Massarelli et al., 2006). The different response in potato and Arabidopsis is not surprising, since salt stress responsive genes may have different temporal expression patterns. Wang et al. (2003) were able to distinguish 39 different expression kinetics profiles for salt stress responsive genes. Only 5 of the 472 analyzed salt stressinduced genes showed a pattern similar to the potato ASG1. The difference in expression pattern of Asg1 in the two analyzed species may be associated to species specificities and/or to the different developmental stages considered (adult hydroponically grown plants of potato and liquid cultured seedlings of Arabidopsis).

Further analysis of Asg1 gene expression profile in response to exogenous stimuli in Arabidopsis revealed that Asg1 is also induced by treatments with other salts $(\mathrm{LiCl}, \mathrm{KCl})$ and by $\mathrm{ABA}$, while it was not responsive to treatments with other hormones such as auxins (IAA) or cytokinins (BAP) (data not shown). Taken together, these results indicate that Asg1 expression might be regulated through a pathway dependent of ABA, a major secondary messenger that regulates the expression of large sets of stress responsive genes (Yamaguchi-Shinozaki and Shinozaki, 2006). The tissue specific expression of Asg1 in Arabidopsis was also investigated. Asg1 promoter analysis showed that this gene is widely expressed in Arabidopsis adult plants and seedlings. GUS expression driven by the Asg1 promoter was particularly high in vascular leaf veins, stems and the abscission and apical zone of siliques. The presence of GUS activity in pollen grains probably reflects that this tissue is developmentally programmed for desiccation tolerance, hence prone to regulate genes responsive to stress (Villalobos et al., 2004).

Additional information about Asg1 function was gathered by analyzing the sub-cellular compartmentalization of the encoded protein. Using onion cells, we found that the Asg1-GFP-derived fluorescence has a non-continuous pattern close to the plasma membrane. A large body of evidence supports the existence, in cell membranes, of functional microdomains or lipid rafts (Brown and London, 1998) which are supposed to play important roles in protein sorting, signal transduction, and cold acclimation (Simons and Ikonen, 1997; Rietveld and Simons, 1998; Minami et al., 2010). In plants, lipid rafts were associated with proteins involved in signaling and response to biotic and abiotic stress, cellular trafficking 


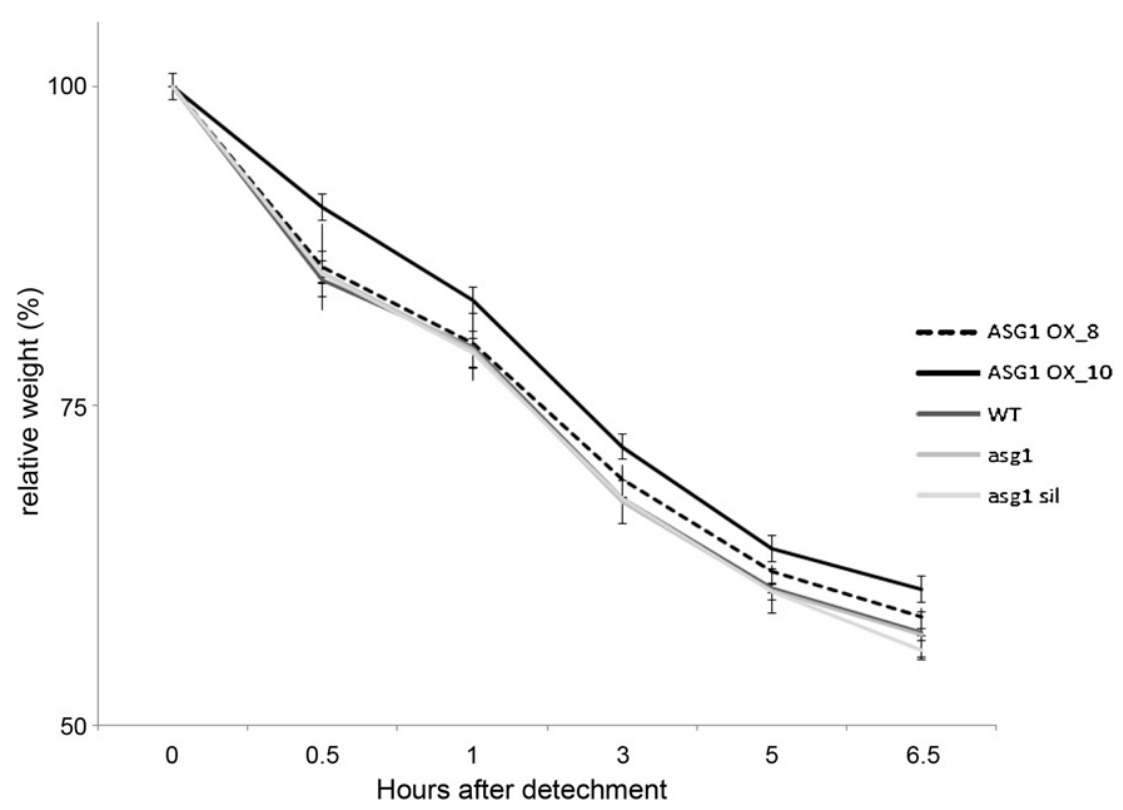

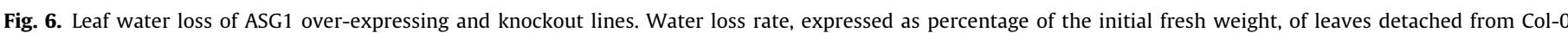

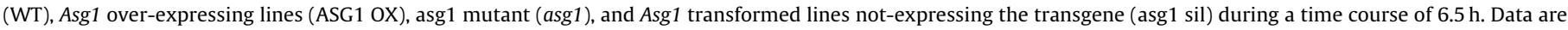
means \pm SD of six replicates.

and cell wall metabolism, and are likely to constitute, as in animal cells, signaling platforms involved in the mentioned physiological functions (Morel et al., 2006; Mongrand et al., 2010). The peculiar localization of the Asg1-GFP fusion protein observed using onion cells raises the interesting hypothesis that ASG1 may be part of such membrane microdomains. Further experiments, however, are needed to confirm this hypothesis.

\section{Asg1 is a functional determinant of salt stress adaptation}

Functional analysis of Asg1 in potato and Arabidopsis was further carried out by generating transgenic potato and Arabidopsis plants over-expressing Asg1. In addition, Asg1 knockout mutants of Arabidopsis (asg1) were obtained for loss-of-function studies. Overexpression of Asg1 amplifies stress responses typically observed during stress adaptation (Nakashima et al., 2009; Zou et al., 2011). Indeed, potato Asg1 over-expressing lines exposed to long-term salt stress had a significantly higher stomatal resistance compared to their relative controls, while no differences in productive parameters (Leaf Area and tubers Fresh Weight), in salt or untreated plants, were detected. Higher stomatal resistance with no decrease in leaf area or yield is typically considered a desirable trait to increasing Water Use Efficiency (Yoo et al., 2009). Stomata closure is among the earliest responses to hyper-osmotic stress, since it consents to control tissue water content and cellular turgor, which is necessary to warrant growth even at a much slower rate (Munns and Tester, 2008). While the coordination of these events is mostly under hormonal control and leads to a new homeostatic asset for water and ions (Barragán et al., 2012), the efficiency of these processes determines the degree of adaptation. As matter of fact, stomata closure will preserve plants from wilting in the short-term but in the longterm it will arrest plant growth since it will restrict $\mathrm{CO}_{2}$ uptake. With the exception of one line, which had a significantly lower water potential probably due to a delayed timing of stomata closure in that specific experiment, the higher stomatal resistance of Asg1 transgenic plants did not significantly affect the plant water potential, which was similarly reduced in both transgenic and control plants in response to salinity. Surprisingly, the remarkably increased stomatal resistance of Asg1 overexpressing plants did not affect plant growth in the presence of salt as it would have been expected because of a restricted availability of $\mathrm{CO}_{2}$ for carboxylation reactions. At $100 \mathrm{mM} \mathrm{NaCl}$, the leaf area of $15 \mathrm{~A} 8$ was even slightly higher compared to both wild type and vector controls. Tuber yield, however, was not improved in Asg1 over-expressing plants. Consistent with most published literature (Bressan et al., 2009), manipulation of Asg1 expression did not improve yield. This is not surprising due to the complexity of responses triggered by abiotic stresses and the number of players participating in these processes. Furthermore, in potato the production does not simply coincide with aboveground dry mass but it involves growth and development of storage organs.

The over-expression of Asg1 in Arabidopsis gave similar results to those observed in potato. The water loss rate of leaves detached from Asg1 over-expressing plants was slightly reduced compared to control plants. asg1 mutant did not show significant differences compared to the wild-type in terms of water loss rate indicating that ASG1 is not required for proper stomatal closure in regulation of water loss but rather its function may be redundant with other proteins.

Based on the results of Asg1 over-expression studies in potato and Arabidopsis, it can be concluded that an over-expression of Asg1 leads to a more efficient stomatal closure upon osmotic stress. Stomatal closure upon dehydration as well as germination in stress conditions are controlled in large part by ABA (Xiong and Zhu, 2003; Ruggiero et al., 2004; Raghavendra et al., 2010). Indeed, many key genes involved in ABA biosynthesis, signaling, or ABA-dependent transcription regulation were initially identified by screening of mutants with altered stomatal behaviors or seed germination in stress conditions (Leung et al., 1997; Mustilli et al., 2002; Kuhn et al., 2006). Phenotypes similar to those observed in plants with altered expression of Asg1 were reported, for example, in Arabidopsis plants over-expressing two ABF type transcription factors which displayed a reduced transpiration as well as glucose and salt hyper-sensitive germination (Kang et al., 2002). 

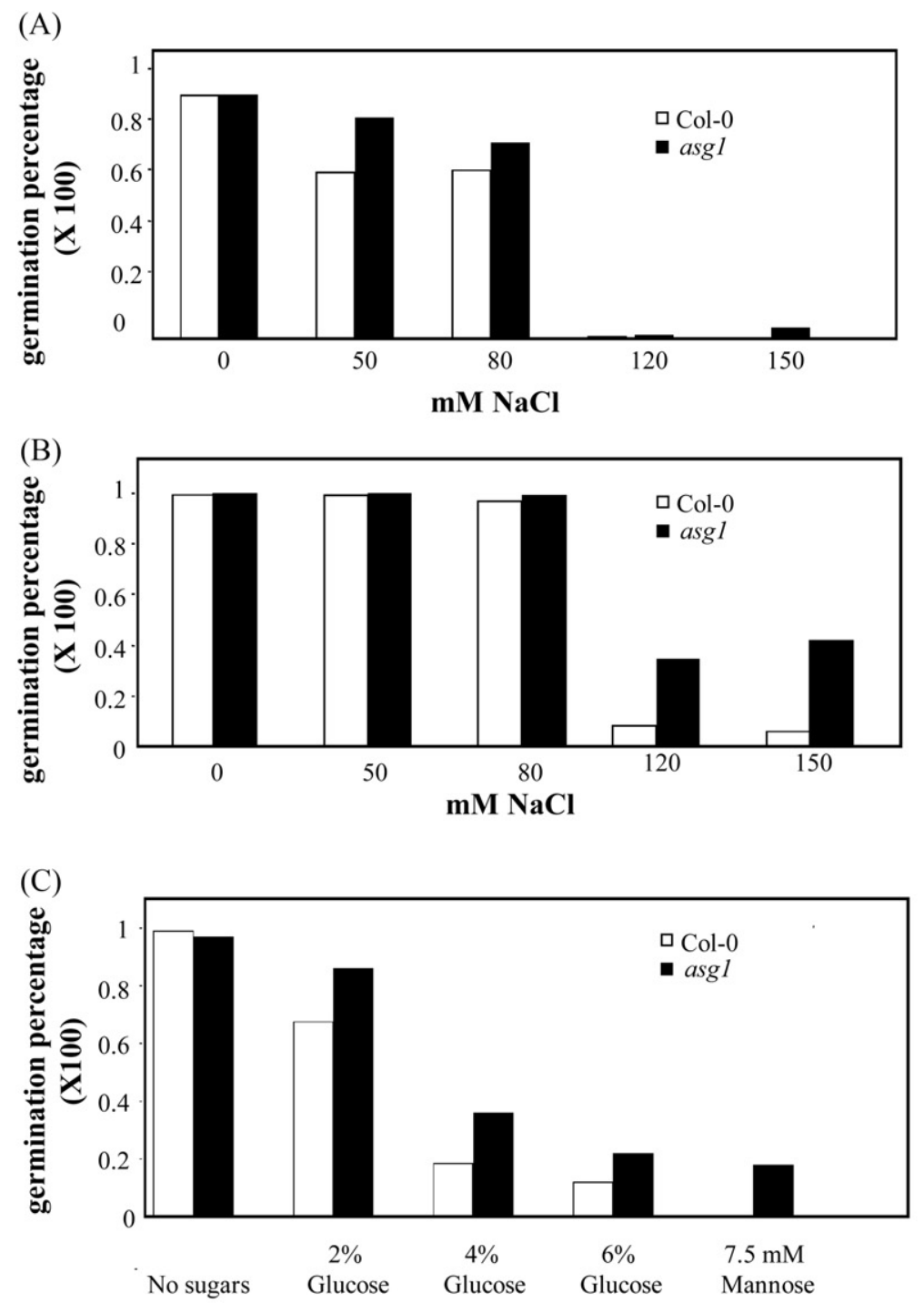

(D)

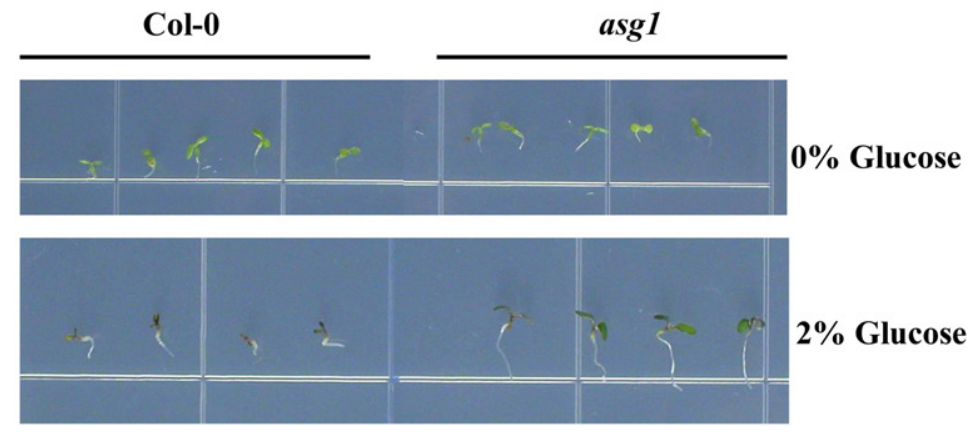

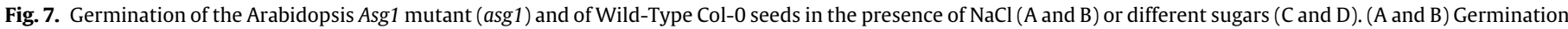

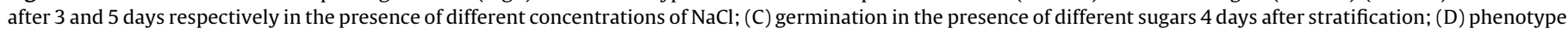
of seedlings of asg1 and Col-0 grown in the presence of $2 \%$ Glucose. Germination was scored in terms of radical emergence.

Conversely, loss-of-function mutations in positive regulators of ABA response caused a salt- and glucose-insensitive germination (Chen et al., 2008; Söderman et al., 2000). Although at present a biological function to proteins encoded by Asg1 genes could not be assigned, phenotype similarities of Asg1 over-expressors and loss-of-function mutants with known positive regulators of ABA response suggest that Asg1 may be involved in ABA-mediated response to salt and osmotic stresses. Further experiments will be necessary to prove this hypothesis.

\section{Acknowledgments}

The authors are grateful to Becky Stevenson and Lorenza Sannino for excellent technical assistance and to the Arabidopsis Biological Resource Center for providing the Arabidopsis T-DNA insertion mutant. GB was supported by a Ph.D. fellowship from the University of Naples Federico II. This work was funded by MIUR project FIRB Plant-STRESS to SG.

Contribution no. 374 of CNR-IGV. 


\section{Appendix A. Supplementary data}

Supplementary data associated with this article can be found, in the online version, at http://dx.doi.org/10.1016/j.jplph.2012.07.004.

\section{References}

Amtmann A, Leigh R. Ion homeostasis. In: Pareek A, Sopory SK, Bohnert HJ, editors. Abiotic stress adaptation in plants. Dordrecht, The Netherlands: Springer; 2010. p. 245-62.

Arenas-Huertero F, Arroyo A, Zhou L, Sheen J, León P. Analysis of Arabidopsis glucose insensitive mutants, gin 5 and gin6, reveals a central role of the plant hormone ABA in the regulation of plant vegetative development by sugar. Genes Dev 2000;14:2085-96.

Aroca R, Porcel R, Ruiz-Lozano JM. Regulation of root water uptake under abiotic stress conditions. J Exp Bot 2012;63:43-57.

Atkinson NJ, Urwin PE. The interaction of plant biotic and abiotic stresses: from genes to the field. J Exp Bot 2012., http://dx.doi.org/10.1093/jxb/ers100.

Barragán V, Leidi EO, Andrés Z, Rubio L, De Luca A, Fernandez JA, et al. Ion Exchangers NHX1 and NHX2 Mediate Active Potassium Uptake into Vacuoles to Regulate Cell Turgor and Stomatal Function in Arabidopsis Plant Cell 2012;24:1127-42.

Bressan R, Bohnert H, Zhu J-K. Abiotic stress tolerance: from gene discovery in model organisms to crop improvement. Mol Plant 2009;2:1-2.

Brown DA, London E. Functions of lipid rafts in biological membranes. Annu Rev Cell Dev Biol 1998;14:111-36.

Chen J-Q, Meng X-P, Zhang Y, Xia M, Wang X-P. Over-expression of OsDREB genes lead to enhanced drought tolerance in rice. Biotechnol Lett 2008;30:2191-8.

Chołuj D, Karwowska R, Ciszewska A, Jasińska M. Influence of long-term drought stress on osmolyte accumulation in sugar beet (Beta vulgaris L.) plants. Acta Physiol Plant 2008;30:679-87.

Clough SJ, Bent AF. Floral dip: a simplified method for Agrobacterium-mediated transformation of Arabidopsis thaliana. Plant J 1998;16:735-43.

Curtis MD, Grossniklaus U. A gateway cloning vector set for high-throughput functional analysis of genes in planta. Plant Physiol 2003;133:462-9.

Davis SJ, Vierstra RD. Soluble, highly fluorescent variants of green fluorescent protein (GFP) for use in higher plants. Plant Mol Biol 1998;36:521-8.

De Palma M, Grillo S, Massarelli I, Costa A, Balogh G, Vigh L, et al. Regulation of desaturase gene expression, changes in membrane lipid composition and freezing tolerance in potato plants. Mol Breeding 2008;21:15-26.

Deikman J, Petracek M, Heard JE. Drought tolerance through biotechnology: improving translation from the laboratory to farmers' fields. Curr Opin Biotechnol 2012;23:243-50.

Flowers TJ. Improving crop salt tolerance. J Exp Bot 2004;55:307-19.

Foyer $\mathrm{CH}$, Shigeoka S. Understanding oxidative stress and antioxidant functions to enhance photosynthesis. Plant Physiol 2011;155:93-100.

Fujita T, Maggio A, Garcia-Rios M, Bressan RA, Csonka LN. Comparative analysis of the regulation of expression and structures of two evolutionarily divergent genes for Delta1-pyrroline-5-carboxylate synthetase from tomato. Plant Physiol 1998;118:661-74.

Kang J-Y, Choi H-I, Im M-Y, Kim SY. Arabidopsis basic leucine zipper proteins that mediate stress-responsive abscisic acid signaling. Plant Cell 2002;14:343-57.

Karaba A, Dixit S, Greco R, Aharoni A, Trijatmiko KR, Marsch-Martinez N, et al. Improvement of water use efficiency in rice by expression of hardy, an arabidopsis drought and salt tolerance gene. Proc Natl Acad Sci USA 2007;104:15270-5.

Klingler JP, Batelli G, Zhu J-K. ABA receptors: the START of a new paradigm in phytohormone signalling. J Exp Bot 2010;61:3199-210.

Krasensky J, Jonak C. Drought, salt, and temperature stress-induced metabolic rearrangements and regulatory networks. J Exp Bot 2012;63:1593-608.

Kuhn JM, Boisson-Dernier A, Dizon MB, Maktabi MH, Schroeder JI. The protein phosphatase AtPP2CA negatively regulates abscisic acid signal transduction in Arabidopsis, and effects of abh1 on AtPP2CA mRNA. Plant Physiol 2006;140:127-39.

Kuromori T, Miyaji T, Yabuuchi H, Shimizu H, Sugimoto E, Kamiya A, et al. ABC transporter AtABCG25 is involved in abscisic acid transport and responses. Proc Natl Acad Sci USA 2010;107:2361-6.

Lee SC, Luan S. ABA signal transduction at the crossroad of biotic and abiotic stress responses. Plant Cell Environ 2012;35:53-60.

Leung J, Merlot S, Giraudat J. The Arabidopsis ABSCISIC ACID-INSENSITIVE2 (ABI2) and $\mathrm{ABI} 1$ genes encode homologous protein phosphatases $2 \mathrm{C}$ involved in abscisic acid signal transduction. Plant Cell 1997:9:759-71.

Massarelli I, Cioffi R, Batelli G, De Palma M, Costa A, Grillo S, et al. Functional screening of plant stress-related cDNAs by random over-expression in Escherichia coli. Plant Sci 2006;170:880-8.

Minami A, Furuto A, Uemura M. Dynamic compositional changes of detergentresistant plasma membrane microdomains during plant cold acclimation. Plant Signal Behav 2010;5:1115-8.

Mongrand S, Stanislas T, Bayer EMF, Lherminier J, Simon-Plas F. Membrane rafts in plant cells. Trends Plant Sci 2010;15:656-63.
Morel J, Claverol S, Mongrand S, Furt F, Fromentin J, Bessoule JJ, et al. Proteomics of plant detergent-resistant membranes. Mol Cell Proteomics 2006;5:1396411.

Munns R. Comparative physiology of salt and water stress. Plant Cell Environ 2002;25:239-50.

Munns R, Tester M. Mechanisms of salinity tolerance. Annu Rev Plant Biol 2008;59:651-81.

Murashige T, Skoog F. A revised medium for rapid growth and bio assays with tobacco tissue cultures. Physiol Plant 1962;15:473-97.

Mustilli A-C, Merlot S, Vavasseur A, Fenzi F, Giraudat J. Arabidopsis OST1 protein kinase mediates the regulation of stomatal aperture by abscisic acid and acts upstream of reactive oxygen species production. Plant Cell 2002;14:308999.

Nakashima K, Ito Y, Yamaguchi-Shinozaki K. Transcriptional regulatory networks in response to abiotic stresses in arabidopsis and grasses. Plant Physiol 2009;149:88-95.

Nelson DE, Repetti PP, Adams TR, Creelman RA, Wu J, Warner DC, et al. Plant nuclear factor $\mathrm{Y}(\mathrm{NF}-\mathrm{Y}) \mathrm{B}$ subunits confer drought tolerance and lead to improved corn yields on water-limited acres. Proc Natl Acad Sci USA 2007;104:16450-5.

Pang Q, Hays JB, Rajagopal I. Two cDNAs from the plant Arabidopsis thaliana that partially restore recombination proficiency and DNA-damage resistance to E. coli mutants lacking recombination-intermediate-resolution activities. Nucleic Acids Res 1993;21:1647-53.

Pardo JM. Biotechnology of water and salinity stress tolerance. Curr Opin Biotechnol 2010;21:185-96.

Qin F, Shinozaki K, Yamaguchi-Shinozaki K. Achievements and challenges in understanding plant abiotic stress responses and tolerance. Plant Cell Physiol 2011;52:1569-82.

Raghavendra AS, Gonugunta VK, Christmann A, Grill E. ABA perception and signalling. Trends Plant Sci 2010;15:395-401.

Rietveld A, Simons K. The differential miscibility of lipids as the basis for the formation of functional membrane rafts. Biochim Biophys Acta 1998;1376:467-79.

Ruggiero B, Koiwa H, Manabe Y, Quist TM, Inan G, Saccardo F, et al. Uncoupling the effects of abscisic acid on plant growth and water relations. analysis of sto1/nced3, an abscisic acid-deficient but salt stress-tolerant mutant in arabidopsis. Plant Physiol 2004;136:3134-47.

Schardl CL, Byrd AD, Benzion G, Altschuler MA, Hildebrand DF, Hunt AG. Design and construction of a versatile system for the expression of foreign genes in plants. Gene 1987;61:1-11.

Shinozaki K, Yamaguchi-Shinozaki K. Gene networks involved in drought stress response and tolerance. J Exp Bot 2007;58:221-7.

Simons K, Ikonen E. Functional rafts in cell membranes. Nature 1997;387:569-72.

Singh J, Kaur L. Advances in potato chemistry and technology. Academic Press; 2009. p. 508.

Söderman EM, Brocard IM, Lynch TJ, Finkelstein RR. Regulation and function of the Arabidopsis ABA-insensitive4 gene in seed and abscisic acid response signaling networks. Plant Physiol 2000;124:1752-65.

Sunkar R, Kapoor A, Zhu J-K. Posttranscriptional induction of two $\mathrm{Cu} / \mathrm{Zn}$ superoxide dismutase genes in Arabidopsis is mediated by downregulation of miR398 and important for oxidative stress tolerance. Plant Cell 2006;18:2051-65.

Tsugane K, Kobayashi K, Niwa Y, Ohba Y, Wada K, Kobayashi H. A recessive Arabidopsis mutant that grows photoautotrophically under salt stress shows enhanced active oxygen detoxification. Plant Cell 1999;11:1195-206.

Verslues PE, Agarwal M, Katiyar-Agarwal S, Zhu J, Zhu JK. Methods and concepts in quantifying resistance to drought, salt and freezing, abiotic stresses that affect plant water status. Plant J 2006;45:523-39.

Verslues PE, Juenger TE. Drought, metabolites, and Arabidopsis natural variation: a promising combination for understanding adaptation to water-limited environments. Curr Opin Plant Biol 2011;14:240-5.

Villalobos MA, Bartels D, Iturriaga G. Stress tolerance and glucose insensitive phenotypes in Arabidopsis overexpressing the CpMYB10 transcription factor gene. Plant Physiol 2004;135:309-24.

Wang H, Miyazaki S, Kawai K, Deyholos M, Galbraith DW, Bohnert HJ, et al. Temporal progression of gene expression responses to salt shock in maize roots. Plant Mol Biol 2003;52:873-91.

Weiner JJ, Peterson FC, Volkman BF, Cutler SR. Structural and functional insights into core ABA signaling. Curr Opin Plant Biol 2010;13:495-502.

Xiong L, Zhu J-K. Regulation of abscisic acid biosynthesis. Plant Physiol 2003;133:29-36.

Yamada A, Tsutsumi K, Tanimoto S, Ozeki Y. Plant RelA/SpoT homolog confers salt tolerance in Escherichia coli and Saccharomyces cerevisiae. Plant Cell Physiol 2003;44:3-9.

Yamaguchi-Shinozaki K, Shinozaki K. Transcriptional regulatory networks in cellular responses and tolerance to dehydration and cold stresses. Annu Rev Plant Biol 2006;57:781-803.

Yoo CY, Pence HE, Hasegawa PM, Mickelbart MV. Regulation of transpiration to improve crop water use. CRC Crit Rev Plant Sci 2009;28:410-31.

Zhu J-K. Salt and drought stress signal transduction in plants. Annu Rev Plant Biol 2002;53:247-73.

Zou C, Sun K, Mackaluso JD, Seddon AE, Jin R, Thomashow MF, et al. Cis-regulatory code of stress-responsive transcription in Arabidopsis thaliana. Proc Natl Acad Sci USA 2011;108:14992-7. 